\title{
Literatura y cultura para una didáctica intercultural del español como lengua extranjera (ELE) ${ }^{1}$
}

\section{Literature and Culture for an Intercultural Didactics of Spanish as a Foreign Language (SFL)}

\author{
Noelia Ibarra-Rius \\ Department of Language and Literature Pedagogy, University of Valencia, \\ Av. dels Tarongers, 4, 46022 Valencia, Spain \\ e-mail: Noelia.Ibarra@uv.es \\ Josep Ballester-Roca \\ Department of Language and Literature Pedagogy, University of Valencia, \\ Av. dels Tarongers, 4, 46022 Valencia, Spain \\ e-mail: Josep.Ballester@uv.es
}

\begin{abstract}
This work evolves around a main purpose: to analyze the role of literature in the pedagogy of Spanish as a Foreign Language from an intercultural perspective. From this standpoint, we reflect about the importance of the term Spanish Literature to defend the different literatures implied in the syntagm; hence, we study how comparative literature becomes the main tool to understand linguistic, cultural and literary diversity. We consider that these two ideas should be in the pedagogical profile of the Spanish as a Foreign Language professor.
\end{abstract}

Keywords: literature, culture, Spanish as a foreign language, plurilingual and pluricultural competence

\section{INTRODUCCIÓN}

El destierro padecido por la literatura en la didáctica del español como lengua extranjera se constata todavía en nuestros días a través de una de sus más drásticas

\footnotetext{
${ }^{1}$ Este trabajo se enmarca en el proyecto I+D "Imágenes literarias de la diversidad: ciudadanía e identidad a través de la educación lectora y literaria" (GV2015/050).
} 
consecuencias: su olvido casi sistemático en gran parte de manuales o de materiales de ELE, y en consecuencia, de numerosas programaciones de aula, o en el mejor de los supuestos, su inclusión ya como elemento lúdico respecto a los contenidos clave, ya a manera de recurso de uso exclusivo para el desarrollo de actividades de carácter lingüístico. En cualquier caso, tanto su exilio como las supuestas variantes de su admisión en forma de componente secundario -bien como entretenimiento respecto a contenidos gramaticales, bien como soporte para la adquisición de destrezas de mayor relevancia-, suponen en definitiva, diferentes máscaras de un mismo problema: la creación de un déficit insoslayable en el aprendiz de español.

La didáctica de ELE no puede abordarse desde una perspectiva monolítica que excluya la cultura(s) y la literatura(s), como tampoco puede aspirarse a que el aprendiz adquiera la competencia plurilingüe y pluricultural propugnada por el Marco común europeo de referencia para las lenguas (MCERL) sin tener contacto con una de las manifestaciones lingüísticas y culturales clave como es la literatura. Además, en el caso de ELE cobra todavía más importancia si cabe, ya que el término "español" engloba una gran diversidad de culturas y literaturas a las que el estudiante puede aproximarse a través del texto literario.

Por este motivo, en los últimos tiempos, cada vez más voces reclaman la revisión del estatus del texto literario en el aula de ELE (Sitman \& Lerner, 1994, 1999; Stembert, 2009 [1999]; Naranjo Pita, 1999; Martínez Sallés, 1999, 2004; Sanz Pastor, 2000, 2004; Mendoza, 2004 o Garrido \& Montesa, 2010) y de determinados prejuicios reiterados hasta su conversión en argumentos para justificar su ausencia (Quintana, 1993; Mendoza, 1993; Montesa \& Garrido, 1994; Sanz González, 1995; Pedraza, 1998; García Naranjo \& Moreno García, 2001; Jurado \& Zayas, 2002; Acquaroni, 2007; Albaladejo, 2007; García Parejo, 2009; Peragón, 2011).

Sin embargo, pese a la incontrovertible necesidad de su presencia y al creciente número de especialistas que demandan la perspectiva integradora -lengua, literatura y cultura - como paradigma desde el que abordar la didáctica de ELE, la literatura sigue siendo esa gran olvidada en demasiadas clases, programaciones y materiales.

\section{LITERATURA Y DIDÁCTICA DEL ESPAÑOL COMO LENGUA EXTRANJERA}

La formulación del título de este apartado, a través de la reunión de dos elementos mediante la conjunción copulativa, supone ya una toma de postura explícita $\mathrm{y}$, al tiempo, condensa todo un recorrido por los últimos 60 años de la enseñanza de ELE que culmina en nuestros días con la clausura -al menos, desde la perspectiva teórica- de la controversia sobre la pertinencia de la literatura en el seno del aula de ELE. De hecho, si en lugar de partir del nexo entre ambos componentes hubiéramos optado por su formulación a través de un interrogante (¿literatura en el aula de ELE?), 
habríamos encontrado diferentes respuestas en función del paradigma protagonista. Así, como muestra podemos recordar la restrictiva concepción de la literatura como modelo de lengua del enfoque gramatical, el olvido absoluto sufrido a la luz del auge del modelo estructuralista o el interés que su estudio ha despertado en el seno de las actuales tendencias de la didáctica de las lenguas y las literaturas, basadas en el enfoque comunicativo.

En este sentido, las diferentes consideraciones por las que ha pasado la literatura en la enseñanza/aprendizaje de ELE han sido descritas de forma lúcida por Quintana (1993) en términos de un movimiento pendular cuya trayectoria hacia la inclusión se constata en torno a los años noventa: "si hasta finales de la década de los sesenta la actitud general era de rechazo a la inclusión de textos literarios en los syllabus, creo que actualmente el péndulo se escora hacia el otro lado" (p. 92).

Esta metáfora del trayecto pendular se ha reiterado en la bibliografía reciente como una sintética explicación de todo un recorrido por la enseñanza de ELE estructurado desde la óptica histórica como una sucesión de modelos y, en la que además, se ha acuñado otra imagen: la del esperado retorno de la literatura hasta casi convertirla en un lugar común en gran número de trabajos. Este regreso se enmarca en la perspectiva textual, por lo que "no se habla tanto de literatura como de textos literarios” (Martínez Sallés, 1999, p. 19), entendidos como recursos útiles para la enseñanza y aprendizaje de una lengua extranjera dada su característica de textos auténticos (Martínez Sallés, 2004; Mendoza 2004).

El interés despertado por su estudio, plasmado entre otros aspectos en el creciente número de contribuciones en forma de comunicaciones y ponencias en congresos, trabajos de final de máster, artículos, capítulos de libro y monografías en torno a la temática, nos permite considerarla como una prolífica línea de investigación e innovación docente en la que confluyen nuevas aportaciones con algunas voces ya clásicas (Quintana, 1993; Mendoza, 1993, 2004; Montesa \& Garrido, 1994; Sitman \& Lerner, 1994, 1999; Sanz González, 1995; Pedraza, 1998; Stembert, 2009 [1999]; Naranjo Pita, 1999; Martínez Sallés, 1999, 2004; Martín Perís, 2000; Sanz Pastor, 2000, 2004; García Naranjo \& Moreno García, 2001; Jurado \& Zayas, 2002; Acquaroni, 2007; Albaladejo, 2007; García Parejo, 2009; Garrido \& Montesa, 2010; Peragón, 2011; Núñez, 2015).

Desde nuestra perspectiva, una de las principales consecuencias del incremento de aportaciones estriba en la aparente aceptación de la inclusión de la literatura en ELE por parte de la comunidad académica y docente. De ahí que al inicio de estas páginas apostáramos por el fin de la controversia, al menos desde la perspectiva teórica. Sin embargo, la praxis todavía plantea diferentes problemas en términos de concreción, ya que aún no podemos hablar de un significativo protagonismo de la literatura per se en las actividades cotidianas de formación lingüístico comunicativa, como tampoco en los manuales (Martín Perís, 2000) y, en consecuencia, en las aulas (Albaladejo, 2007). De hecho, en numerosas ocasiones, su presencia responde a la 
motivación individual del docente y, sobre todo, a su concepción de la literatura y del por qué y para qué de su presencia.

Por este motivo, compartimos el pronóstico de Montesa (2012) respecto al regreso, calificado por éste como "inacabado ya que, aunque en el principio todos estamos de acuerdo, en la práctica hay muchas dudas y desorientación" (p. 31). En efecto, nos encontramos ante una compleja paradoja, alimentada por diferentes prejuicios nacidos al compás de las distintas etapas y paradigmas que han permitido la explicación histórica de su destierro, su posición subsidiaria o su restricción a términos utilitarios.

\subsection{CONCEPCIONES Y PREJUICIOS}

Más de dos décadas han transcurrido respecto al dictamen de Montesa \& Garrido (1994) sobre el papel del texto literario "frecuentemente considerándolo de escasa rentabilidad o solamente utilizable con niveles superiores y, por supuesto, como un adorno, como un lujo, como la guinda de un pastel que no pierde nada sin ella" (p. 452), pero la tesis esgrimida continúa repitiéndose con igual fuerza en nuestros días. En el seno de esta concepción subyace una serie de argumentos en los que si profundizamos, nos encontramos en realidad ante un reducido pero poderoso elenco de prejuicios planteados como única concepción válida e irrebatible por su naturalización y frecuencia.

De hecho, creemos que una de las herencias de la didáctica de ELE respecto a la literatura procede de la disociación entre lengua y literatura que ha presidido diferentes modelos de enseñanza vinculados a la lengua materna, pese a la clara interrelación entre aprendizajes lingüísticos y literarios marcada desde los postulados comunicativos de la didáctica de las lenguas y las literaturas contemporánea (Cassany, 1993; Mendoza, 2004; Ibarra-Rius \& Ballester-Roca, 2014; Ballester-Roca, 2015). Por este motivo, creemos fundamental partir de la revisión de tales argumentos y, en consecuencia, de la denuncia de los prejuicios académicos que enmascaran, procedentes en gran número de ocasiones de tradiciones previas y de modelos de enseñanza de la lengua materna.

En primer lugar, uno de los más habituales estriba en la atribución de un elevado grado de dificultad, dado el desvío respecto a la norma que el texto literario entraña. Sin embargo, el texto literario no constituye un sinónimo de la complejidad lingüística, como tampoco necesariamente debe suponer un desvío del uso de la lengua, ya que en gran número de ocasiones, se caracteriza por su sencillez, claridad compositiva y gramatical (Mendoza, 2004; Montesa, 2012).

Desde nuestra perspectiva, el problema no descansaría por tanto, en la naturaleza del texto literario, sino en la selección que de éste se realiza. Por esto, si admitimos 
la dificultad de los textos elegidos como criterio válido, deberíamos extrapolarlo a otro tipo de textos empleados en aulas y manuales a los que no se aplica (Montesa \& Garrido, 1994; Pedraza, 1998; Stembert, 2009 [1999]; Mendoza, 1999; Albaladejo, 2007; Ibarra-Rius \& Ballester-Roca, 2014; Ballester, 2015) y abordar también el cuestionamiento del nivel de complejidad de las tareas programadas en relación con el nivel del discente (Sanz Pastor, 2000).

En esencia, el argumento de la peculiaridad respecto a la norma como justificación de su escasa rentabilidad en actividades cotidianas de formación lingüísticocomunicativa y su exclusión de las premisas esenciales de un aprendizaje pragmático y funcional (Mendoza, 2004; Garrido \& Montesa, 2010) esconde la perspectiva contraria: su extraordinario potencial para la apreciación del uso de diferentes manifestaciones de la diversidad expresiva (Sitman \& Lerner, 1994). Pensemos además que el estudiante no siempre cuenta con oportunidades de exposición directa a la lengua meta, por lo que la literatura permite la ampliación de su abanico de experiencias a través de "un medio alternativo al contacto directo con la lengua que tienen los nativos o los extranjeros en un medio de inmersión" (Montesa \& Garrido, 1994, p. 453). Asimismo, destaca su funcionalidad como enriquecedor input lingüístico y cultural en la formación lingüístico-comunicativa del alumno (Montesa \& Garrido, 1999; Albaladejo, 2004; Martínez Sallés, 2004; Mendoza, 2004; Montesa, 2012), ya que a partir del texto literario puede observar, interiorizar y sistematizar conocimientos, referentes y aplicaciones normativas, pragmáticas y funcionales.

De este prejuicio parte otro de los más frecuentes: la consideración de la literatura como un material artístico y, por tanto, no auténtico, artificial y descontextualizado de los usos reales de la lengua. No obstante, "la lengua real sólo tiene dos vías para presentarse ante el estudiante: 1) la comunicación espontánea con los nativos [...]; 2) la lectura de textos literarios" (Pedraza, 1998, p. 61). Además, los textos literarios presentan la cualidad de la autenticidad en la misma medida que otros textos empleados en el aula, con la ventaja añadida de la significatividad frente a productos artificiales que figuran en los manuales de ELE en forma de diálogos $a d$ hoc (Montesa, 2012).

Por otra parte, la interpretación de la enseñanza de idiomas en términos propios del capitalismo neoliberal, esto es, como rentabilidad o eficacia en términos de utilidad funcional, supone en definitiva:

dar paso a excesos contrarios en contradicción con el papel que tiene que desarrollar la escuela (excesos que estuvieron de moda en ciertas metodologías, v. gr. en los métodos estructuroglobales). De seguir en esa dirección habría que prescindir también de los textos literarios en la enseñanza de la lengua materna y de cuanto no tiene una utilidad inmediata (Stembert, 2009 [1999], p. 247)

La asunción de este prejuicio implica, asimismo, una noción de competencia comunicativa y de mundo real tan restrictiva que subordina los intereses del apren- 
diente a la satisfacción exclusiva de las necesidades inmediatas. Sin embargo, nuestra experiencia nos demuestra que las exigencias básicas del estudiante dan paso a otras más profundas, vinculadas a la comunicación interpersonal y el desarrollo de la identidad individual que el aprendizaje de una lengua en definitiva, también supone. En este punto "es donde entra la literatura como posibilidad de comprometer de un modo total a la persona en el mensaje" (Montesa \& Garrido, 1994, p. 449), como conjunción de las diferentes destrezas a través de la reflexión personal del lector y del placer estético, lúdico o cognoscitivo derivado de su lectura.

En esta línea, nos encontramos con el último de los argumentos más repetidos: la falta de atractivo del texto literario para el aprendiente de ELE y, de nuevo, su escasa rentabilidad en la consecución de sus objetivos profesionales. Sin embargo, esta consideración alberga diferentes estereotipos: en primer lugar, la concepción del colectivo "aprendiz de ELE" como un todo homogéneo en el que no existen intereses diferenciados; en segundo, se parte del rechazo y desinterés del alumnado como única posibilidad de recepción respecto al texto literario; y, en tercero, la existencia de discrepancias en la aplicación de criterios, pues las preferencias del estudiante no se aplican como criterio de selección a otras posibilidades textuales -canciones, noticias, diálogos ficticios o listas de instrucciones- empleadas en aulas y manuales y que pueden igualmente no interesarle al aprendiz de ELE.

Resulta evidente que la literatura no tiene por qué constituir el centro de interés vital de todo aprendiente de ELE, pero también debemos contemplarla como posibilidad, pues podemos encontrarnos con discípulos cuya recepción de un poema ha sorprendido al profesorado, que solicitan ampliación de información sobre un autor o incluso, del corpus de textos trabajados en el aula (García Naranjo \& Moreno García, 2001; Santamaría Busto, 2012). De hecho, la seducción por la literatura de otra cultura puede convertirse en el estímulo principal para la aproximación a la lengua extranjera (Sanz Pastor, 2006) e incluso despertar en el alumnado "la motivación para leer e incluso mejorar sus destrezas de comprensión lectora, elementos que definitivamente contribuirán a su desempeño como lectores de todo tipo de textos" (Sitman \& Lerner, 1994, p. 227). De esta forma, no solo redundará en la rentabilidad profesional o académica del estudiante, dado que desarrollará su competencia literaria y lectora $-\mathrm{y}$, por tanto, comprenderá de forma autónoma una mayor tipología de textos-, sino que también puede facilitar el life long learning, incluso cuando el curso o el periodo de exposición a la lengua meta ha finalizado.

Por último, apuntamos cómo en realidad los distintos argumentos examinados enmascaran un prejuicio de base al que no siempre se alude de forma directa: el miedo, tanto del aprendiz como del docente respecto al empleo de un material que exige un esfuerzo extra por parte de ambos para su inclusión en el aula. En consecuencia, resulta esencial partir de la superación de este temor como primera estrategia de aproximación al inmenso abanico de posibilidades que el texto literario ofrece y plantear de forma contextualizada su programación didáctica. 


\subsection{LITERATURA SÍ, PERO ¿CÓMO?}

El texto literario supone para el estudiante una aportación singular que no proporciona otra tipología textual: el disfrute estético, lúdico, cognoscitivo o emocional. El lector debe dialogar con el texto para construir el significado de forma autónoma y al tiempo, experimenta el placer de su lectura. En este proceso comunicativo se establece una interacción única entre ambos que conlleva componentes emocionales, afectivos, intelectuales, estéticos y lúdicos, no activos en otro tipo de recursos $\mathrm{y}$ vinculados al desarrollo de las competencias lectora, literaria y comunicativa. Por este motivo, requiere de una didáctica en la que se atienda los diferentes componentes -cognoscitivo lúdico, estético, sentimental y reflexivo- para poder disfrutarlo de forma plena y diferenciada de otras posibilidades textuales (Sanz Pastor, 2006; Ibarra-Rius \& Ballester-Roca, 2014). De esta manera, el texto literario no solo permitirá la adquisición y desarrollo de diferentes destrezas y competencias esenciales como la lectora, literaria y, en definitiva, la comunicativa, sino también la intercultural o social y ciudadana, además del placer y el enriquecimiento personal (Stembert, 2009 [1999]; Mendoza, 1999, 2004; Sanz Pastor, 2000, 2006; Aventín, 2004; Martínez Sallés, 2004; Ambassa, 2006; Montesa, 2012; Ibarra-Rius \& Ballester-Roca, 2014; Ballester, 2015).

En este sentido, compartimos las reticencias de diferentes investigadores respecto a los peligros de despojar a la literatura de sus cualidades inherentes para justificar su inclusión en el aula y, de esta manera, convertirla en otro material más, en un pretexto o en un mero recurso para la enseñanza lingüística, pues creemos con Montesa \& Garrido (1994) que "el texto literario no debe traicionarse" (p. 453). Por este motivo, pensamos que su presencia debería responder a una metodología específica, la didáctica del texto literario en ELE desde una perspectiva intercultural, articulada a partir de dos variables esenciales: los rasgos intrínsecos al texto literario y el alumnado contemporáneo.

Desde nuestra perspectiva, su singularidad genera uno de los mayores problemas respecto a su didáctica, pues nos encontramos ante la ausencia de una reflexión consensuada en torno a los criterios específicos para su programación en ELE. Por esto, cuando en el mejor de los supuestos nos encontramos con una clara voluntad de implantar la literatura en el aula de ELE, en numerosas ocasiones este deseo se estrella ante la disparidad y dispersión de propuestas para concretarla, unida al creciente número de interrogantes que no encuentran una respuesta unívoca en la crítica especializada.

Así, la primera duda radica en los propios objetivos didácticos, es decir, qué vamos a hacer con el texto literario o para qué vamos a utilizarlo: ¿optaremos por privilegiar la función estética, incidiremos en contenidos lingüístico-comunicativos, nos centraremos en distinguir los referentes socioculturales? Por nuestra parte, creemos que la respuesta pasa por considerar los tres aspectos anteriores de acuerdo con 
la consecución de los objetivos didácticos comunicativos programados y las posibilidades didácticas que la literatura nos brinda.

A continuación, surge rápidamente el cómo, es decir, con qué textos, cómo seleccionarlos y de qué manera enfocarlos. En este sentido, diferentes autores (Naranjo, 1999; Sanz Pastor, 2006) abogan de forma exclusiva por la obra literaria en detrimento de las adaptaciones, comprendidas como transgresiones de la autenticidad del texto literario. Así, por ejemplo, Albaladejo (2007) considera las adaptaciones como textos simplificados, como “obras literarias a las que se les ha 'amputado' por así decirlo ciertos aspectos considerados complejos y que por ello han perdido la autenticidad del original. Si como en un principio defendíamos el valor de la literatura como material auténtico, ¿por qué entonces utilizar material ‘mutilado’?” (p. 12).

No obstante, desde las aportaciones contemporáneas de la didáctica de las literaturas y las lenguas se han reivindicado las adaptaciones como una opción válida y consensuada entre docentes e investigadores. Así, la adaptación no constituye por sí misma un atentado contra el carácter sacro de la literatura, sino que dependerá, al igual que el mismo texto literario, de su calidad, pues "adaptar tiene que ser un verbo -al igual que 'traducir'- que nada tenga que ver con 'traicionar'; una adaptación no es una versión libre, no es una usurpación -un robo- de personajes para inventarles una nueva vida literaria" (Navarro, 2013, p. 64).

Así, distintos investigadores defienden como inexcusable la lectura de la obra completa con una consecuencia clara: la imposibilidad de articular dinámicas de trabajo en torno a fragmentos de textos literarios, ya que mutilarían el significado de la obra y, por tanto, atentarían contra el desarrollo de las competencias lectora y literaria. Si bien parecen evidentes las ventajas de la lectura de una obra completa para el acceso a su significado global, no creemos encontrarnos ante opciones excluyentes. Nos referimos a que pueden coexistir en el aula ambas estrategias, esto es, la lectura de obras completas y de fragmentos de acuerdo con la finalidad de potenciar el trabajo en torno a una secuencia concreta, o bien podemos abordar la lectura de fragmentos sin cercenar el interés del alumnado por la obra. De esta manera, la explotación de fragmentos literarios puede conducir a una experiencia completa en todos los sentidos, pues desembocaría en la lectura del libro en cuestión.

En esencia, más que de controversias, pensamos que ambas posturas -negación de adaptaciones y fragmentos- coinciden en un planteamiento restrictivo y elitista de la obra literaria. Por este motivo, reivindicamos con Sanz Pastor (2006) la necesaria desacralización del texto literario que nos permita acceder a sus entresijos lingüísticos, creativos y estratégicos desde una mirada respetuosa, pero en absoluto reverencial. Desde nuestra óptica, más que de una dicotomía irreconciliable -obra/adaptación, obra/fragmento- desde la que podríamos abordar otras de semejantes características - ¿textos actuales o clásicos?-, creemos que el planteamiento estriba en la elección del texto literario que se realice. En otros términos, pensamos que el principal problema radica en la configuración del canon de aula por parte del docen- 
te, en su calidad y adecuación para un grupo clase concreto, apuntado por diferentes autores (Cassany, 1993; Montesa \& Garrido, 1994; Sitman \& Lerner, 1994; Pedraza, 1998; Stembert, 2009 [1999]; Mendoza, 1999, 2004; Montesa, 2012; Ibarra-Rius \& Ballester-Roca, 2014; Ballester, 2015). Sin embargo, no queremos presentar el canon de aula con las connotaciones de un problema de compleja resolución, pues para nosotros supone todo lo contrario: un extraordinario elenco de posibilidades de elección para el docente, en el que siempre encontrará una alternativa idónea para cada grupo clase.

\section{LITERATURA, LENGUA Y CULTURA EN ELE}

Entre los diferentes exponentes que nos permiten explicar la necesidad de la literatura en ELE, quizás la clave radique en sus posibilidades didácticas para la educación intercultural y, en este sentido, su papel fundamental en la adquisición y desarrollo de la competencia comunicativa, comprendida desde una perspectiva más amplia que la reproducción de enunciados en determinados contextos comunicativos. Se trata en definitiva, del objetivo apuntado desde el Marco común europeo de referencia para las lenguas: enseñanza, aprendizaje, evaluación (2002): la adquisición de la competencia plurilingüe y pluricultural, como macrocompetencia en la que la cultura adquiere un papel clave.

De esta manera, el aprendizaje de una lengua extranjera constituiría "un ejercicio de comunicación intercultural, una aventura íntima de encuentro $-\mathrm{y}$ a veces de desencuentro- consigo mismo y con los demás" (Sitman \& Lerner, 1999) en el que el texto literario, como "mosaico de la interculturalidad" (Aventín, 2004), construye "un lugar de encuentro con las ideas del otro en un ámbito no tópico" (Montesa $\&$ Garrido, 1994, p. 455). En este espacio generado por la literatura puede gestarse la competencia intercultural que permite desarrollar las distintas facetas comunicativas de la LE (Ibarra-Rius \& Ballester-Roca, 2014), como la posibilidad de interpretación de informaciones que remitan a un marco cultural diferente del propio (Sitman \& Lerner, 1994).

De hecho, la literatura constituye la manifestación cultural más representativa de una lengua, "una inestimable fuente de información cultural -en el doble sentido del mundo representado y de la propia obra como elemento de la cultura" (Sanz Pastor, 2006, p. 124). Por este motivo, la literatura implica una extraordinaria oportunidad de aproximación a la cultura de la lengua meta y a la propia lengua meta, pues permite: a) proporcionar información cultural explícita; b) inferir información cultural en sí misma; c) reflejar particularidades discursivas propias de la lengua meta y, por supuesto, d) es un producto cultural en sí mismo (Acquaroni, 2007).

Por esto, los textos literarios constituyen un motivador recurso para la aceptación de la diversidad sociocultural (Mendoza, 2004) desde el que articular un en- 
riquecedor elenco de actividades para el desarrollo tanto de aspectos genéricos relativos al aprendizaje lingüístico y cultural como facetas específicas para el aprendizaje comunicativo, sin que esta afirmación implique en modo alguno su uso o abuso como pretexto exclusivo para la realización de ejercicios. En esta línea, la presencia del texto literario en ELE requiere de la asunción por parte del docente de su importancia para la adquisición del componente sociocultural y la influencia de este en el desarrollo del aprendizaje de la lengua (Sanz Pastor, 2010).

La relevancia de la competencia plurilingüe y pluricultural como base para el desarrollo de una verdadera competencia comunicativa en el aprendizaje de una lengua extranjera implica en definitiva, la introducción de una perspectiva integradora configurada por tres ejes: lengua, literatura y cultura, defendida por numerosas voces como imprescindible en la actual didáctica de ELE (Sitman \& Lerner, 1999; García Naranjo \& Moreno García, 2001; García Parejo, 2009; Sanz Pastor, 2010 o Peragón, 2011, entre otros). Frente a concepciones reduccionistas en torno al papel de la literatura en la didáctica de las lenguas extranjeras, nos posicionamos en la más contemporánea que comprende su importancia no solo como un recurso más, sino como un instrumento privilegiado para la adquisición y desarrollo de las diferentes competencias, la comunicativa, la discursiva, la léxico-gramatical, la sociocultural -por supuesto-, la emocional, la literaria y la lectora y, sobre todo, la plurilingüe y pluricultural defendida desde el MCERL.

\subsection{CANON, LITERATURAS Y CULTURAS}

Si la literatura supone un espacio clave para la aproximación a la cultura y esta es por definición necesariamente diversa, su integración en el aula de ELE nos permitirá desarrollar la competencia intercultural desde la que trascender la falsa homogeneidad oculta tras términos como "literatura o cultura española", pues, ¿a qué cultura(s) o a qué literatura(s) nos estamos refiriendo? La reflexión en torno al conjunto de obras comprendidas en este sintagma nominal nos desvela su configuración a partir del calificativo que acompaña al sustantivo "literatura o cultura" y que en ocasiones, se elige en función de la nacionalidad atribuida al texto o, en su defecto, de la lengua que representa.

Sin embargo, este criterio de clasificación genera problemáticas atribuciones, cuya consecuencia más drástica radica en la homogeneización del rico patrimonio cultural, lingüístico y literario que engloba tal sintagma. En primer lugar, recordemos que, al hablar de ELE, el referente cultural comprende no solo el ámbito español sino también el hispanoamericano, pese a que no siempre se contemplen ambos. Desde esta óptica, la tarea del profesor de ELE responde a su función como mediador tanto del idioma como del país o países a cuya cultura y literatura atañe, una suerte de embajador en este caso, no solo del ámbito peninsular, sino de todo el 
hispanoamericano (Sitman \& Lerner, 1994). En segundo lugar, afecta a la supuesta unicidad del término "cultura" o "literatura", pues obvia las diferentes culturas y literaturas, de gran riqueza y diversidad entre sí que coexisten en su seno, y que en el caso del Estado español presentan sustanciales diferencias. Por último, esta homogeneización implica la reiteración de tópicos y estereotipos culturales (King, 1999; Areizaga, 2002; Miquel \& Sans, 2004; Paricio, 2005) que han presidido gran parte de los manuales de ELE de décadas anteriores y, por tanto, condicionado la mirada del discente de ELE y su interpretación del referente "español".

Parece evidente que la defensa de la inclusión de la literatura en ELE no implica su didáctica en términos de adquisición de conocimientos memorísticos o filológicos y menos de todo el elenco de obras canónicas de la denominada "literatura española" (Ibarra-Rius \& Ballester-Roca, 2014), ya que no corresponde al objetivo esencial de su programación. Asimismo, deberíamos definir muy claramente qué entendemos por esta y, en este sentido, qué autores deberían configurarla, pues nos encontraríamos con casos fronterizos para esta etiqueta como Vázquez Montalbán o Manuel Vicent, entre otros muchos ejemplos de escritores que oscilan entre dos identidades culturales (un escritor catalán que escribe en lengua española en el primer caso y un escritor valenciano cuya producción es también en lengua española en el segundo). Podríamos proseguir la ejemplificación de autores escindidos entre dos atribuciones culturales y enmarcados en una determinada categoría en función de la lengua predominante de escritura en detrimento de su aparición en otras clasificaciones vinculadas, por ejemplo, al lugar de nacimiento y viceversa, por lo que el interrogante formulado hace décadas de King (1999) nos parece de total vigencia a este respecto:

a la hora de enseñar la cultura española plantea preguntas y dudas sobre qué cultura se debe enseñar. Y si existen varias culturas españolas, ¿cómo puede el profesor de E/LE enseñar esta diversidad sin limitarse exclusivamente, como hace Mesonero Romanos, a las expresiones culturales de una sola región, sean, por ejemplo, de Castilla, Galicia o Andalucía?

Nuestra respuesta para que el alumno se aproxime a esta pluralidad cultural y lingüística y trascienda posibles prejuicios desde manifestaciones culturales reales de indudable calidad radica en la literatura y en el comparatismo como metodología idónea (Mendoza, 2004; Ibarra-Rius \& Ballester-Roca, 2014; Ballester, 2015).

El comparatismo en la enseñanza de ELE permite numerosas posibilidades para la configuración de un canon de aula de calidad que respete los intereses, necesidades y niveles de los estudiantes sin necesidad de obedecer de forma ciega las listas configuradas de acuerdo con etiquetas como "movimiento literario", "época histórica", "pertenencia geográfica", "lengua de escritura" o incluso "género". En el seno de una didáctica de ELE intercultural, el comparatismo implica una valiosa herramienta desde la que erradicar prejuicios y ofrecer cánones de aula que combinen 
autores, géneros, lenguas y nacionalidades en función de temas, tópicos o personajes desde los que plantearse la universalidad de estos y potenciar la aproximación a otras culturas a través del diálogo, el debate y la reflexión individual y colectiva.

\section{CONCLUSIONES}

La literatura, como manifestación única de la cultura y de la lengua meta, supone un material auténtico irreemplazable en el aula de ELE para la adquisición de la competencia plurilingüe y pluricultural, así como para competencias clave como la lectora, la literaria, la comunicativa o la emocional (Sanz Pastor, 2006), pero también, y sobre todo, para la comprensión y aprehensión de realidades culturales diferentes a la propia, la lectura crítica de la realidad circundante, la aproximación al otro, la socialización e, incluso, el descubrimiento de uno mismo. Tal y como afirma un destacado teórico con el que nos gustaría cerrar estas páginas, la literatura:

amplía nuestro universo, nos incita a concebir otras maneras de concebirlo y de organizarlo [...] expande hasta el infinito esta posibilidad de interacción con los otros y, por tanto, nos enriquece infinitamente. Nos proporciona sensaciones irreemplazables que hacen que el mundo real sea más bello y tenga más sentido. Lejos de ser un simple añadido, una distracción reservada a las personas educadas, permite que cada uno responda mejor a su vocación de ser Humano. (Todorov, 2009, p. 15) 


\section{BIBLIOGRAFÍA}

Acquaroni, R. (2007). Las palabras que no se lleva el viento: literatura y enseñanza de español como LE/L2. Madrid: Santillana.

Albaladejo, M. D. (2007). Cómo llevar la literatura al aula de ELE: de la teoría a la práctica. MarcoELE, 5. Disponible en http://marcoele.com/descargas/5/albaladejo-literaturaalaula.pdf

Ambassa, C. (2006). Algunas preocupaciones didácticas y metodológicas en la enseñanza de literatura en clase de ELE. RedELE, 8. Disponible en http://www.mecd.gob.es/dctm/redele/ Material-RedEle/Revista/2006_08/2006_redELE_8_01Ambassa.pdf?documentId=0901e72b $80 \mathrm{df} 365 \mathrm{e}$

Areizaga, E. (2002). El componente cultural en la enseñanza de lenguas: elementos para el análisis y la evaluación del material didáctico. Cultura y Educación, 14 (2), 161-175.

Aventín, A. (2004). Del contexto al texto: El texto literario en ELE. Reflexiones y propuestas. RedELE, 9.

Ballester-Roca, J. (2015). La formación lectora y literaria. Barcelona: Graó.

Cassany, D., Luna, M. \& Sanz, G. (1993). Ensenyar llengua. Barcelona: Graó.

Consejo de Europa (2002). Marco común europeo de referencia para las lenguas: aprendizaje, enseñanza, evaluación. Madrid: Secretaría General Técnica MEC, Anaya \& Instituto Cervantes. Disponible en http://cvc.cervantes.es/ensenanza/biblioteca_ele/marco

García Naranjo, F. \& Moreno García, C. (2001). Cuentos, cuentos, cuentos. Variación y norma en la presentación de un texto literario. En: M. A. Martín Zorraquino \& C. Díez Pelegrín (Eds.), ¿Qué español enseñar? Norma y variación lingüística en la enseñanza del español como lengua extranjera (pp. 819-829). Zaragoza: Universidad de Zaragoza.

Garrido, A. \& Montesa, S. (2010). La recuperación de la literatura en la enseñanza del ELE. Una necesidad y una propuesta. MarcoELE, 11. Disponible en http://marcoele.com/descargas/ navas/19.garrido-montesa.pdf

Ibarra-Rius, N. \& Ballester-Roca, J. (2014). ¿Crónica de un divorcio anunciado: la enseñanza de la literatura y la lectura en el siglo XXI. En: M. Campos \& E. Martos (Coords.), Cartografias lectoras y otros estudios de lectura (pp. 189-200). Madrid: Marcial Pons.

Jurado, J. \& Zayas, F. (2002). La literatura en la enseñanza del español como lengua extranjera. Cádiz: Servicio de Publicaciones Universidad de Cádiz.

King, S. (1999). Desempeñar papeles y la desmitificación cultural en El amante bilingüe de Juan Marsé. Journal of Iberian and Latin American Studies, 5 (2), 73-85.

Martín Peris, E. (2000). Textos literarios y manuales de enseñanza de español como lengua extranjera. Lenguaje y Textos, 16, 101-131.

Martínez Sallés, M. (1999). Los retos pendientes de la literatura en ELE. Mosaico, 2, 19-22.

- (2004). Libro, déjame libre. Acercarse a la literatura con todos los sentidos, RedELE, 0. Disponible en http://www.mecd.gob.es/dctm/redele/Material-RedEle/Revista/2004_00/2004_red ELE_0_19Martinez-Salles.pdf?documentId=0901e72b80e0c9e4

Mendoza, A. (1993). Literatura, cultura, intercultura. Reflexiones didácticas para la enseñanza de español lengua extranjera. Lenguaje y Textos, 3, 19-42.

- (2004). Los materiales literarios en la enseñanza de ELE: funciones y proyección comunicativa. RedELE, 1. Disponible en http://www.mecd.gob.es/dctm/redele/Material-RedEle/Revista/ 2004_01/2004_redELE_1_07Mendoza.pdf?documentId=0901e72b80e06817

Miquel, L. \& Sans, N. (2004). El componente cultural: un ingrediente más en las clases de lengua. RedEle, 0. Disponible en http://www.mecd.gob.es/dctm/redele/Material-RedEle/Revista/2004 _00/2004_redELE_0_22Miquel.pdf?documentId=0901e72b80e0c8d9

Montesa, S. (2012). Lengua y literatura. Un camino de ida y vuelta. En: J. de Santiago Guervós, H. Bongaerts, J. J. Sánchez Iglesias \& M. Seseña Gómez (Eds.), Del texto a la lengua: La aplicación de los textos a la enseñanza-aprendizaje del español L2-LE (vol. 1, pp. 29-45). Sa- 
lamanca: ASELE. Disponible en http://cvc.cervantes.es/ensenanza/biblioteca_ele/asele/pdf/ 21/21_0029.pdf

Montesa, S. \& Garrido, A. (1994). La literatura en clase de lengua. En: S. Montesa \& S. Garrido (Eds.), Actas del II Congreso Internacional de ASELE (1990). Español para extranjeros: didáctica e investigación (pp. 449-457). Málaga: Universidad de Málaga.

Naranjo Pita, M. (1999). La poesía como instrumento didáctico en la clase de español como lengua extranjera. Madrid: Edinumen.

Navarro, R. (2013). La salvación de los clásicos: las adaptaciones fieles al original. Quaderns de Filologia. Estudis literaris, XVIII, 63-75.

Núñez, X. (2015). La didáctica de la literatura en la enseñanza de ELE. Monográfico Lenguaje $y$ Textos, 42, 5-71.

Paricio, M. S. (2005). La dimensión cultural en los libros de texto de lenguas extranjeras: pautas para su análisis. Glosas didácticas, 15, 133-144.

Pedraza, F. (1998). La literatura en la clase de español para extranjeros. En: A. Celis \& J. R. Heredia (Eds.), Lengua y cultura en la enseñanza del español a extranjeros. Actas del VII Congreso de ASELE (pp. 59-66). Cuenca: Universidad de Castilla-La Mancha. Disponible en http://cvc.cervantes.es/ensenanza/biblioteca_ele/asele/pdf/07/07_0057.pdf

Peragón, C. E. (2011). Los textos literarios más que un recurso: análisis de materiales recientes para la enseñanza de la Literatura Española a extranjeros. RedEle, 11.

Pérez Parejo, R. (2009). Modelos de mundo socioculturales en la historia de la literatura española (Automatización y descodificación para alumnos de E/LE). Trujillo \& Miajadas: Junta de Extremadura (Tejuelo. Didáctica de la lengua y la literatura. Monográfico).

Quintana, E. (1993). Literatura y enseñanza de E/LE. En A. Garrido \& S. Montesa (Eds.), Actas del III Congreso Nacional de ASELE El español como lengua extranjera. De la teoría al aula. (pp. 89-92). Málaga: ASELE. Disponible en http://cvc.cervantes.es/ensenza/biblioteca_ele/ asele/pdf/03_0087.pdf

Santamaría Busto, E. (2012). Didáctica de la poesía: estudio y propuestas metodológicas para la explotación de textos poéticos en el aula de ELE. Didáctica, 24, 433-472.

Sanz González, F. (1995). La literatura en clase de lengua extranjera: ¿una presencia incómoda? Didáctica, 7, 119-132.

Sanz Pastor, M. (2000). La literatura en el aula de ELE. Frecuencia-L, 14, $24-27$.

- (2004). Principios de creatividad y teoría literaria aplicados a la enseñanza de E/LE. El símbolo: conceptos generales y repercusiones didácticas. Mosaico, 13.

- (2006). Didáctica de la literatura: el contexto en el texto y el texto en el contexto. Carabela, 59, 5-24.

- (2010). La construcción del componente cultural: enfoque comunicativo y literatura. MarcoELE, 10, 129-138.

Sitman, R. \& Lerner, I. (1994). Literatura hispanoamericana: herramienta de acercamiento cultural en la enseñanza del español como lengua extranjera. En S. Montesa \& P. Gomis (Eds.), Tendencias actuales en la enseñanza del español como lengua extranjera. Actas del V Congreso Internacional de ASELE (pp. 227-233). Málaga: ASELE. Disponible en http://cvc.cervantes. es/ensenanza/biblioteca_ele/asele/pdf/05/05_0225.pdf

- (1999). La literatura del mundo hispanohablante en el aula de E/LE: ¿Un lugar de encuentro o desencuentro? Espéculo, 12. Disponible en http://www.ucm.es/info/especulo/numero12/ cbelatxt.html

Stembert, R. (2009 [1999]). Propuestas didácticas de los textos literarios en la clase de E/LE. MarcoELE, 9, 247-265. Disponible en http://marcoele.com/descargas/expolingua_1999. stembert.pdf

Todorov, T. (2009). La literatura en peligro. Barcelona: Galaxia Gutenberg. 